\title{
Investigation on the Unsteady Hydrodynamic Loads of Ship Passing by Bridge Piers by a 3-D Boundary Element Method
}

\author{
Liang $\mathrm{Li}^{1}$, Zhi-Ming Yuan ${ }^{1}$, Chunyan $\mathrm{Ji}^{2}$,*, Ming-Xin $\mathrm{Li}^{1}$, Yan $\mathrm{Gao}^{1}$ \\ ${ }^{I}$ Department of Naval Architecture, Ocean and Marine Engineering, University of Strathclyde, 100 Montrose \\ Street, Glasgow, G4 OLZ, UK \\ ${ }^{2}$ School of Naval Architecture and Ocean Engineering, Jiangsu University of Science and Technology, Zhenjiang, \\ 212003, China \\ *Corresponding author: jichunyanjkd@163.com
}

\begin{abstract}
A 3-D Rankine type Green function boundary element method is developed to estimate the unsteady hydrodynamic interaction during ship passing piers process. To address this moving boundary problem, a free surface re-meshing algorithm based on the combination of local mesh and global mesh is proposed to update fluid boundary and the boundary value formula is solved at each time step. Two alternative numerical models for ship passing piers problem are compared with the current analysis method. Based on the three numerical models, the characteristics of the hydrodynamic loads acting on the passing ship are specified and the critical positions for the peak values have been identified. Simulation results demonstrate that the fluid disturbance induced by the piers is of importance. Therefore, the study of ship-piers hydrodynamic interaction in a river must be handled as a different forward speeds problem. Additionally, the wave elevation effect can only be neglected on condition that the forward speed is very low.
\end{abstract}

Keywords: boundary element method; Rankine source; ship-to-piers; unsteady hydrodynamic interaction; passing and encountering

\section{Introduction}

The unsteady fluid interaction between two moving bodies during a passing by process has long been investigated, mainly in terms of aerodynamics and hydrodynamics. Based on 3-D 
compressible Euler/Naiver-Stokes equations, Fujii and Ogawa [1] simulated the flow filed induced by two trains passing by each other in a tunnel. The basic characteristics of the time history of pressure distributions and the aerodynamic forces were identified. Mancini and Malfatti [2] performed a full-scale field measurement of the unsteady aerodynamic pressure generated by a train passing at high speed in open air and in tunnels. Watanabe and Matsuno [3] investigated the flows driven by a high-speed car passing through a hairpin corner. A so-called 'Moving Computational Domain Method' was proposed and the whole computational domain including bodies inside moved in the physical space without the limit of region size. Yeung and Tan [4] studied the hydrodynamic interaction of ships with fixed obstacle. The slender-body theory was used with the assumption that the free surface was rigid. Kijima [5] investigated the ship-piers interaction in close proximity with the asymptotic expansions method. Time series of wave forces and ship motions were obtained.

Research on multi-body hydrodynamic interaction during passing by process was firstly started decades ago with model test approach. Newton [6] carried out model test of two ships during overtaking operations in deep water. Müller [7] studied overtaking and encountering problem in restricted water channel. Vantorre et al. [8] carried out extensive model tests on the hydrodynamic interaction between two ships during overtaking, passing by, and encountering operations. A specially designed twin-carriage system was applied to implement the complicated operations. Model test program was launched by Mousaviraad et al. [9] to investigate ship-to-ship interactions in the calm water and waves during overtaking operations. Effect of configuration, speed and heading angle were studied.

Alongside with model test method, analytical and empirical approaches mostly based on the slender-body theory have been developed and validated. Tuck and Newman [10] extended the slender-body theory to predict the hydrodynamic lateral force and yaw moment acting on each of the two ships while they were moving along parallel paths. Yeung [11] used similar approach to investigate the unsteady hydrodynamic interaction of two ships moving in shallow water. Brix [12] proposed an approximate empirical formula to estimate the maximum values of longitudinal and transverse forces during overtaking operations. Wang [13] expanded Yeung's [11] study to investigated the irrotational flow around two slender bodies with revolution angles of yaw, which were travelling along parallel paths in close proximity. 
More recently, the boundary element method is applied in an increasing number of numerical studies on unsteady hydrodynamic interaction within the framework of potential flow theory. Xiang and Faltinsen [14] developed a 3-D Rankine source method with consideration of the linear wave effect to solve the boundary value problem of two ships advancing in waves. Sutulo et al. [15] developed a potential-flow method to estimate the hydrodynamic interaction forces. The general estimation method was validated against experimental data obtained in deep and shallow water towing tanks for the case of a tug operating near a larger vessel. Yuan et al. [16] proposed a methodology to predict the ship-to-ship interaction during overtaking operations in shallow water. To deal with the different forward speeds, they divided the velocity potential into two independent components and addressed each component separately. Yao and Dong [17] developed a frequency domain analysis method with consideration of local steady flow effect to investigate wave forces of two parallel advancing ships. They showed that the flow speed is an important factor relating to the hydrodynamic interaction. Xu et al. [18] used high-order element method to predict hydrodynamic interaction of two cylindroids moving along parallel courses in shallow water. Since free surface elevation was neglected in their work, only low forward speed problem was addressed. Pinkster and Bhawsinka [19] proposed a real-time simulation technique for ship-ship interaction based on a double-body flow method. Wang and Zou [20] studied the hydrodynamic interaction between a passing ship and a berthed ship in a single way lock.

The hydrodynamic interaction involved in the ship passing piers process has not been fully studied due to its complexity. The speed of river flow could make the coordinate system complicated. The bridge piers cannot be treated as fixed obstacles and the 'passing by' process turns to an encountering problem, which is a different forward speeds problem by nature. Also, the ship usually passes by the piers with a moderate or even high forward speed so that the wave elevation effect must be considered. This study aims to address these issues involved in the ship passing piers problem. A 3-D boundary element method based on Rankine type Green function is developed. Since it is an unsteady and moving boundary problem, a re-meshing algorithm is developed to update boundary of fluid domain at each time step. To fully capture the free surface disturbance effect, the Neumann-Kelvin condition is applied on the free water surface. The current analysis method will be compared with another two numerical models to illustrate the wave elevation effect 
and the importance of fluid disturbance induced by the piers in river flow.

\section{Mathematical formulations}

Theoretically, ship passing pier (involving relative movement) is an unsteady problem, since the boundary of the fluid domain is time-varying. Nevertheless, it can be handled in a quasi-steady approach. The steady boundary value model is set up at each time step, based on the current fluid boundary configuration. The boundary value problem is solved in a step-by-step manner with the update of boundary configuration to consider the unsteady effect.

\subsection{Boundary value problem}

For a single ship advancing in open calm water with constant forward speed $u$, a velocity potential $\varphi$ is introduced. Following [21], the dynamic free surface condition and the kinetic free surface condition can be expressed as

$$
\begin{gathered}
u \frac{\partial \varphi}{\partial x}+\frac{1}{2} \nabla \varphi \cdot \nabla \varphi+g \xi=0, \text { on } z=\xi(x, y) \\
u \frac{\partial \xi}{\partial x}-\frac{\partial \varphi}{\partial z}+\frac{\partial \varphi}{\partial x} \cdot \frac{\partial \xi}{\partial x}+\frac{\partial \varphi}{\partial y} \cdot \frac{\partial \xi}{\partial y}=0, \text { on } z=\xi(x, y)
\end{gathered}
$$

where $\xi$ is the wave elevation. Neglecting higher order terms, the classical linear Neumann-Kelvin free surface condition is derived

$$
u^{2} \frac{\partial^{2} \varphi}{\partial x^{2}}+g \frac{\partial \varphi}{\partial z}=0, \text { on } z=0
$$

Assume that a ship is passing by piers with constant forward speed $u$ and the river flow speed is $u_{0}$. A river-fixed coordinate system moving together with the river flow is introduced. In the riverfixed coordinate, both the passing ship and the piers are moving with forward speed $u_{1}=u-u_{0}$ and $u_{2}=-u_{0}$, respectively.

Theoretically, it is a body-to-body encountering problem. Nevertheless, it is commonly simplified and described with Eq. (4), where the flow potential induced by the pier is neglected. The main reason of such simplification lies in the speed-dependent term in the Neumann-Kelvin condition, which will cause difficulties in a different forward speeds problem. The simplified model inherently neglects the fluid disturbance induced by the piers although the wave elevation effect is considered. However, no single academic study has been undertaken to check the feasibility of this simplified model. 


$$
\begin{aligned}
& \nabla^{2} \varphi=0, \text { in fluid domain } \\
& \frac{\partial \varphi}{\partial \boldsymbol{n}}=u_{1} \cdot n_{1}, \text { on passing ship } \\
& \frac{\partial \varphi}{\partial \boldsymbol{n}}=0, \text { on piers } \\
& u_{1}^{2} \frac{\partial^{2} \varphi}{\partial x^{2}}+g \frac{\partial \varphi}{\partial z}=0, \text { on } z=0 \\
& \frac{\partial \varphi}{\partial \boldsymbol{n}}=0, \text { on } z=-H \\
& \varphi=0, \sqrt{x^{2}+y^{2}} \rightarrow \infty
\end{aligned}
$$

where $\boldsymbol{n}=\left(n_{1}, n_{2}, n_{3}\right)$ is the unit normal vector inward on the body surface and $H$ is the water depth.

Another simplification of this problem is the rigid surface model, which neglects the free water surface disturbance. In this way, the speed-dependent terms in the free surface condition are omitted. The boundary value model of the rigid surface model is given as

$$
\begin{aligned}
& \nabla^{2} \varphi=0, \text { fluid domain } \\
& \frac{\partial \varphi}{\partial \boldsymbol{n}}=u_{1} \cdot n_{1}, \text { on passing ship } \\
& \frac{\partial \varphi}{\partial \boldsymbol{n}}=u_{2} \cdot n_{1} \text {, on piers } \\
& \frac{\partial \varphi}{\partial z}=0, \text { on } z=0 \\
& \frac{\partial \varphi}{\partial \boldsymbol{n}}=0, \text { on } z=-H \\
& \varphi=0, \sqrt{x^{2}+y^{2}} \rightarrow \infty
\end{aligned}
$$

Eq. (5) is the so-called rigid surface model used by a majority of researchers in their studies $[10,11,13,18]$. Using the rigid surface model, only a single boundary value equation needs to be established regardless of the number of bodies involved and it avoids the different forward speeds problem in this way. However, the wave elevation effect is neglected in the rigid surface model.

The two methods more or less neglect some aspects of the problem, due to the speed terms in the Neumann-Kelvin condition. To deal with the different forward speeds problem, Yuan et al. [16] proposed an approach, which divides the potential $\varphi$ into two components

$$
\varphi=\varphi_{1}+\varphi_{2}
$$

where $\varphi_{1}$ is the potential induced by the passing ship moving with speed $u_{1}$ while the piers are stationary. $\varphi_{2}$ is the potential induced by the piers moving with speed $u_{2}$ while the passing ship is stationary. The boundary value problems for $\varphi_{1}$ and $\varphi_{2}$ are represented with Eq. (7) and Eq. (8), respectively. 


$$
\begin{aligned}
& \nabla^{2} \varphi_{1}=0, \text { in fluid domain } \\
& \frac{\partial \varphi_{1}}{\partial \boldsymbol{n}}=u_{1} \cdot n_{1} \text {, on passing ship } \\
& \frac{\partial \varphi_{1}}{\partial \boldsymbol{n}}=0, \text { on piers } \\
& u_{1}^{2} \frac{\partial^{2} \varphi_{1}}{\partial x^{2}}+g \frac{\partial \varphi_{1}}{\partial z}=0, \text { on } z=0 \\
& \frac{\partial \varphi_{1}}{\partial \boldsymbol{n}}=0, \text { on } z=-H \\
& \varphi_{1}=0, \sqrt{x^{2}+y^{2}} \rightarrow \infty \\
& \nabla^{2} \varphi_{2}=0, \text { in fluid domain } \\
& \frac{\partial \varphi_{2}}{\partial \boldsymbol{n}}=0, \text { on passing ship } \\
& \frac{\partial \varphi_{2}}{\partial \boldsymbol{n}}=u_{2} \cdot n_{1}, \text { on piers } \\
& u_{2}^{2} \frac{\partial^{2} \varphi_{2}}{\partial x^{2}}+g \frac{\partial \varphi_{2}}{\partial z}=0, \text { on } z=0 \\
& \frac{\partial \varphi_{2}}{\partial \boldsymbol{n}}=0, \text { on } z=-H \\
& \varphi_{2}=0, \sqrt{x^{2}+y^{2}} \rightarrow \infty
\end{aligned}
$$

It is the coupled model to describe the ship passing piers problem, which accounts for both the wave elevation effect and the fluid disturbance induced by the river flow passing the piers. In the coupled model, the fluid disturbance induced by the passing ship and the pier are addressed separately within their own body-fixed coordinate system. Subsequently, the two potential components are combined to represent the hydrodynamic couplings. In the original work of Yuan et al. [16], the coupled model was applied to low speed problem so that the unsteadiness of the hydrodynamic interaction was not considered. Nevertheless, the contribution of the unsteady term to the pressure will be significant in high speed problem. Therefore, the assumption made by Yuan et al. [16] does not held and the unsteady term should be taken into account when calculating the pressure over the ship hull. In the present study, some modifications are made in order to account the unsteadiness raised by the unsteady pressure distributed over the ship hulls, which will be presented in the following part. It is worth noting that the Neumann-Kelvin free surface condition is typically used for slender bodies. Considering that the river flow speed is usually low, it is applicable to the potential induced by the piers.

As well known, the shallow water effect has a significant influence on the hydrodynamic performance of an advancing ship. To focus on the scope of this study, the water depth is set to 30 
Therefore, the shallow water effect is limited in this study.

Ship passing piers is a moving boundary problem by nature. The ship-pier configuration varies during the passing process. As stated in the beginning of this section, a quasi-steady approach is used. A re-meshing algorithm is first developed to update the boundary configuration at each simulation time step. During the simulation process, the boundary value model is set up based on the updated boundary configuration and the velocity potential is calculated step by step. The unsteady effect is considered by the update of the boundary configuration.

Once the velocity potential $\varphi$ is solved, the hydrodynamic pressure on the ship and the piers are obtained from Bernoulli's equation.

$$
\begin{aligned}
& p_{1}(t)=-\rho\left(\frac{\partial \varphi}{\partial t}-u_{1} \frac{\partial \varphi}{\partial x}+\frac{1}{2} \nabla \varphi \cdot \nabla \varphi\right) \\
& p_{2}(t)=-\rho\left(\frac{\partial \varphi}{\partial t}-u_{2} \frac{\partial \varphi}{\partial x}+\frac{1}{2} \nabla \varphi \cdot \nabla \varphi\right)
\end{aligned}
$$

where $\rho$ is the fluid density. The hydrodynamic forces are obtained by integrating hydrodynamic pressure across the wetted surface.

$$
\begin{aligned}
& F_{i}^{1}(t)=\iint_{S_{1}} p_{1}(t) n_{i} d S, i=1,2, . ., 6 \\
& F_{i}^{2}(t)=\iint_{S_{2}} p_{2}(t) n_{i} d S, i=1,2, . ., 6
\end{aligned}
$$

$n_{i}$ is the generalized normal factor defined by Eq. (11). $\boldsymbol{r}=(x, y, z)$ is the position vector.

$$
n_{i}=\left\{\begin{array}{c}
\boldsymbol{n}, i=1,2,3 \\
\boldsymbol{r} \times \boldsymbol{n}, i=4,5,6
\end{array}\right.
$$

Wave elevation is estimated by

$$
\xi(t)=-\frac{1}{g}\left[\frac{\partial \varphi}{\partial t}-u_{1} \frac{\partial \varphi_{1}}{\partial x}-u_{2} \frac{\partial \varphi_{2}}{\partial x}\right]
$$

Compared with the force model in Yuan et al. [16], we make an improvement to consider the unsteady effect by introducing $\frac{\partial \varphi}{\partial t}$ in Eq. (9). It should be noted that the boundary value problem is solved without consideration of the unsteady effects. Therefore, the fully unsteady effects cannot be estimated by the present methodology. A more sophisticated method, which takes the timedependent terms in the free-surface condition, should be proposed to address this issue. Although the unsteady problem is handled with a quasi-steady approach, the unsteadiness is still reflected in the derivative of velocity potential in time scale. In our numerical model, the unsteady term $\frac{\partial \varphi}{\partial t}$ at time step $i$ is calculated by 


$$
\frac{\partial \varphi_{i}}{\partial t}=\left\{\begin{array}{cc}
\frac{\varphi_{i}-0}{d t} & i=1 \\
\frac{\varphi_{i}-\varphi_{i-1}}{d t} & i=2 \\
\frac{1.5 \varphi_{i}-2 \varphi_{i-1}+0.5 \varphi_{i-2}}{d t} & i>2
\end{array}\right.
$$

where $\varphi_{i}$ is the potential at time step $i . d t$ is the simulation time step.

\subsection{Discretization of the boundary integral}

By distributing Rankine type sources on the entire boundary of the fluid domain, the velocity potential $\varphi(\boldsymbol{r})$ at point $\boldsymbol{r}=(x, y, z)$ within the fluid domain is given by Eq. (14).

$$
\varphi(\boldsymbol{r})=\iint_{s} G(\boldsymbol{r}, \boldsymbol{\varsigma}) \cdot \sigma(\varsigma) d S
$$

where $\sigma(\varsigma)$ is the source strength assigned to point $\varsigma=\left(\varsigma_{1}, \varsigma_{2}, \varsigma_{3}\right)$ on the boundary of the fluid domain. $G(r, \varsigma)$ is the Rankine type Green function defined by

$$
G(\boldsymbol{r}, \boldsymbol{\varsigma})=\frac{1}{\sqrt{\left(x-\varsigma_{1}\right)^{2}+\left(y-\varsigma_{2}\right)^{2}+\left(z-\varsigma_{3}\right)^{2}}}
$$

To enhance the computation efficiency, the mirror image approach is applied by treating the river bed as a mirror and Eq. (15) is thus updated as

$$
G(\boldsymbol{r}, \boldsymbol{\varsigma})=\frac{1}{\sqrt{\left(x-\varsigma_{1}\right)^{2}+\left(y-\varsigma_{2}\right)^{2}+\left(z-\varsigma_{3}\right)^{2}}}+\frac{1}{\sqrt{\left(x-\varsigma_{1}\right)^{2}+\left(y-\varsigma_{2}\right)^{2}+\left(z+2 H+\varsigma_{3}\right)^{2}}}
$$

Eq. (14) is an analytical integral formula and should be represented by a discretized approach in the numerical calculation. Dividing the whole boundary into $N$ elements and assuming that the sources in each element are uniformly distributed and possess identical source strength, analytical formula Eq. (14) is expressed in an integral way

$$
\varphi(\boldsymbol{r})=\sum_{j=1}^{N} G_{i j}\left(\boldsymbol{r}, \boldsymbol{S}_{j}\right) \cdot \sigma_{j}
$$

$G_{i j}$ is the influence matrix with dimension $N \times N$, which denotes the potential at point $i$ induced by element $j$ [22]. $\sigma_{j}$ is the source strength assigned to element $j$ and $\boldsymbol{\zeta}_{j}$ is the center of element $j$. Please refer to Hess and Smith [22] for the detailed derivation of $G_{i j}$.

\subsection{Desingularied method}

In principle, the Rankine singularity should be distributed exactly on the boundary of the fluid domain. However, a designularied method has been developed by moving the elements on free water surface a short distance upward. Meanwhile, the collocation points, where boundary condition is 
satisfied, still stay exactly on the free water surface (see Fig. 1). In this study, a raised distance $d z$ $=L_{j} / 10$ suggested by Kim et al. [23] is selected, where $L_{j}$ is the diagonal length of element $j$.

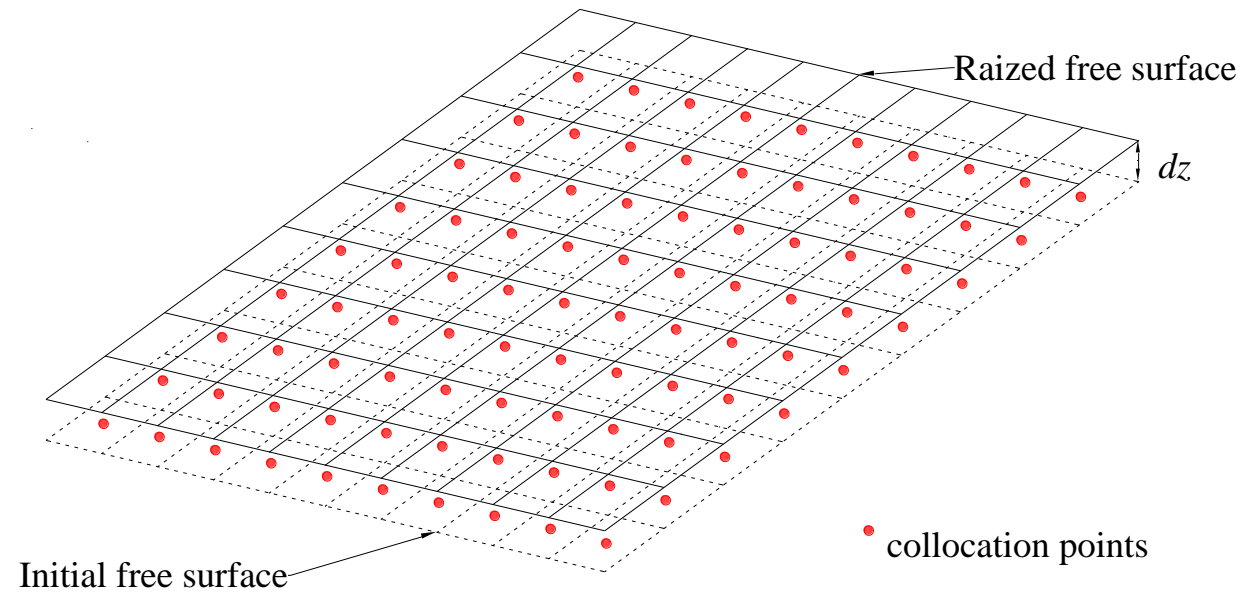

Fig. 1. Raise of the free surface.

The appearance of second derivative of the potential in the free surface condition will cause some difficulties. In theory, second derivative terms can be handled with analytical approach and the exact analytical expression is indeed available. However, it is found that the influence matrix tends to be ill-conditional when analytical representation is applied, which is likely to be caused by sthis problem. They found that the wave pattern developed a saw-toothed appearance and the numerical instability was not caused by rounding errors. Similar problem was reported by $\mathrm{Xu}$ and Yue [24] as well. Although the introduction of a low-pass numerical filter can smooth the wave profile $[24,25]$, there is no evidence showing that this kind of correction approach would not alter the real wave elevation. Therefore, difference scheme rather than analytical formula is applied in this paper to represent the second derivative term. Upwind difference scheme and central difference scheme are two common difference schemes. Generally, the central difference scheme is more accurate while the stability of upwind difference is better. In addition, the application of upwind difference scheme enforces that the wave pattern mainly depends on the upstream flow, which is consistent with physical observation. Due to this favorable property of upwind difference scheme, the second-order upwind difference scheme proposed by Bunnik [26] (see Fig. 2) is adopted to represent the second derivative of velocity potential:

$$
\frac{\partial^{2} \varphi}{\partial x^{2}}\left(\boldsymbol{r}_{i}\right)=\frac{1}{\Delta x_{i}^{2}}\left[\frac{1}{2} \varphi\left(\boldsymbol{r}_{i+4}\right)-2 \varphi\left(\boldsymbol{r}_{i+3}\right)+\frac{11}{2} \varphi\left(\boldsymbol{r}_{i+2}\right)-6 \varphi\left(\boldsymbol{r}_{i+1}\right)+\frac{9}{4} \varphi\left(\boldsymbol{r}_{i}\right)\right]
$$




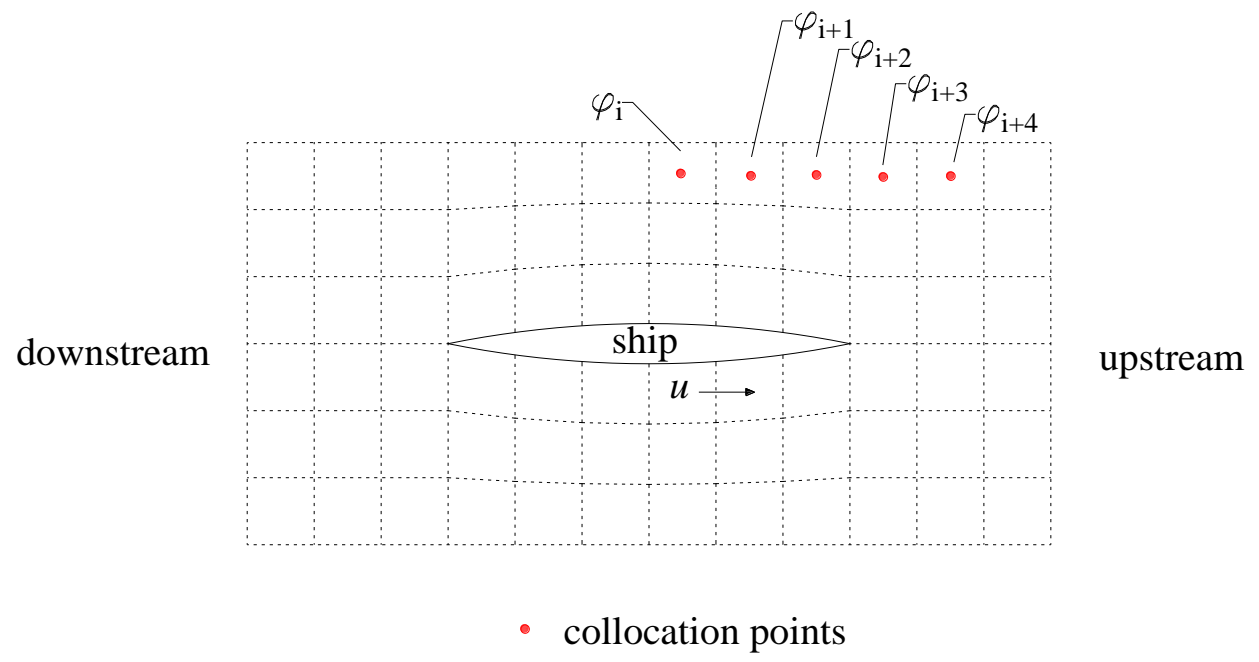

Fig. 2. Second-order upwind difference scheme.

According to $[23,26]$, the radiation condition in the boundary value formula can be satisfied inherently by applying Eq. (18). Therefore, no elements are distributed on the radiation control surface.

\subsection{Re-meshing algorithm}

Ship passing piers is a moving boundary problem by nature. It requires update of the free surface at each time step and the boundary value problem should be solved alongside with the update of element distribution on the free surface. A re-meshing algorithm based on the concepts of local mesh and global mesh is developed. The local mesh is body-fixed and moves together with the passing ship. Comparatively, the global mesh can be understood as a kind of background mesh, which is fixed to the space. The essential idea of the re-meshing algorithm is to use the local mesh to overlap the global mesh and the complicated re-meshing problem will be converted in this way to a simple connection operation. By dividing the entire mesh into two components, the re-meshing algorithm allows the usage of fine elements in local mesh and coarse elements in global mesh.

234 Therefore, it is able to acquire high calculation accuracy with a small amount of elements. Fig. 3 235 displays the fine local mesh, which includes the body surface and the surrounding free water surface. The fine local mesh and coarse global mesh are merged in Fig. 4. 


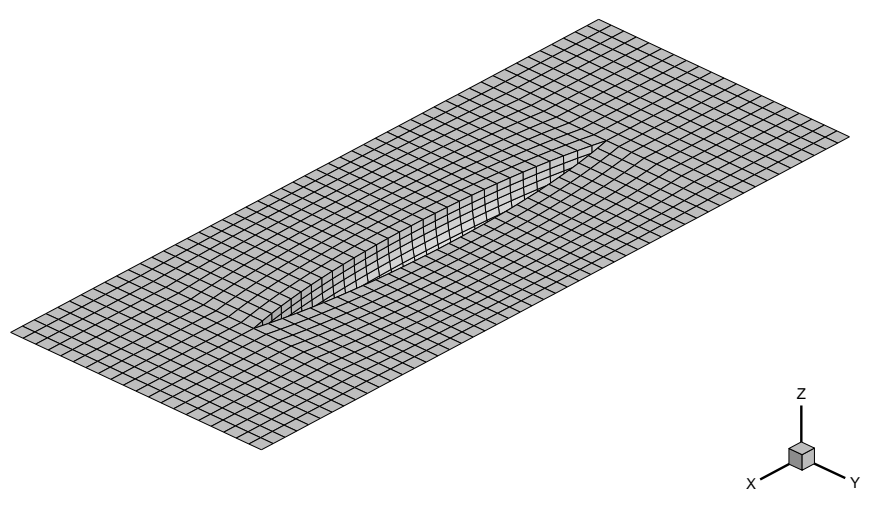

Fig. 3. Local fine mesh.

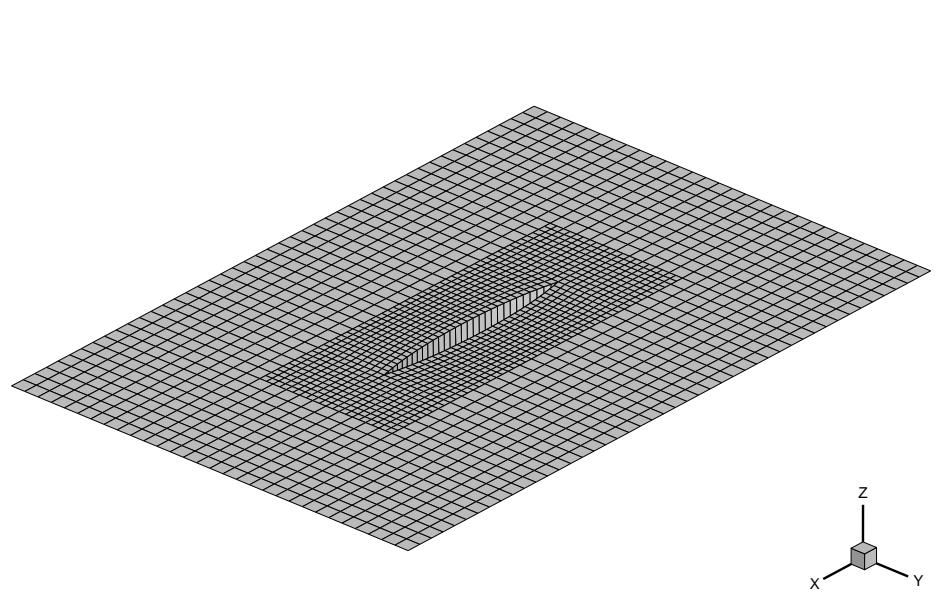

Fig. 4. Merged mesh.

\section{Validation}

Fig. 5 displays the sketch of ship-to-ship operations in close proximity. This section will

243 validate the coupled model against analytical approximation and model test measurement. The

244 hydrodynamic forces presented are normalized by Eq. (19). $L$ is the ship length, $D$ is the draft and $245 \quad B$ is the breath.

$$
\begin{aligned}
C_{x}^{i} & =\frac{F_{x}^{i}}{0.5 \rho u^{2} B D} \\
C_{y}^{i} & =\frac{F_{y}^{i}}{0.5 \rho u^{2} B D} \quad i=1,2 \\
C_{z z}^{i} & =\frac{M_{z z}^{i}}{0.5 \rho u^{2} B D L}
\end{aligned}
$$




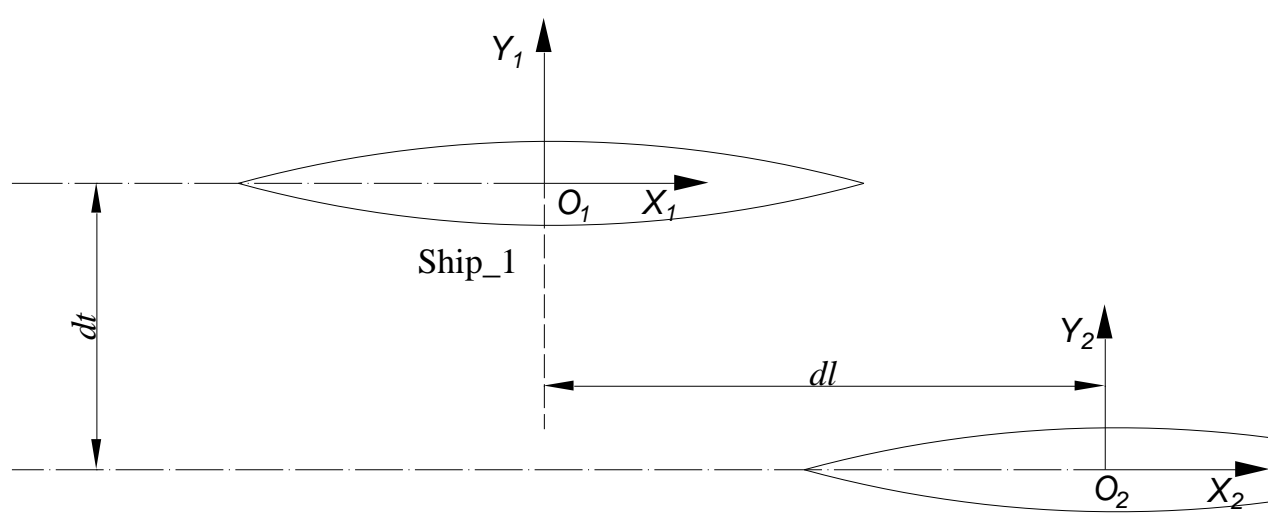

Ship_2

Fig. 5. Sketch of ship-to-ship interaction.

3.1. Validation against analytical approximation

A Wigely ship is set to pass another identical moored Wigely ship in open calm water. The hull geometry is expressed by Eq. (20) with the main dimensions listed in Table 1.

$$
y=\frac{B}{2}\left[1-\left(\frac{z}{D}\right)^{2}\right]\left[1-\left(\frac{2 x}{L}\right)^{2}\right]\left[1+0.2 \cdot\left(\frac{2 x}{L}\right)^{2}\right]
$$

Table 1 Main dimensions of Wigley ship

\begin{tabular}{ll}
\hline Parameter & Value \\
\hline Length $(L)$ & $30 \mathrm{~m}$ \\
Breadth $(B)$ & $3 \mathrm{~m}$ \\
Draft $(D)$ & $1.875 \mathrm{~m}$ \\
\hline
\end{tabular}

Based on the slender-body theory, the following analytical expression is obtained to approximate the hydrodynamic forces acting on the moored ship induced by a passing ship in its proximity [27].

$$
\begin{aligned}
& F_{x}(d l, d t)=\frac{\rho u^{2}}{2 \pi} \int_{L_{1}} A_{2}^{\prime}\left(x_{2}\right) \int_{L_{1}} A_{1}^{\prime}\left(x_{1}\right) \frac{x_{1}-x_{2}+d l}{\left\{\left(x_{1}-x_{2}+d l\right)^{2}+d t^{2}\right\}^{1.5}} d x_{1} d x_{2} \\
& F_{y}(d l, d t)=\frac{\rho u^{2} d t}{\pi} \int_{L_{1}} A_{2}^{\prime}\left(x_{2}\right) \int_{L_{1}} A_{1}^{\prime}\left(x_{1}\right) \frac{1}{\left\{\left(x_{1}-x_{2}+d l\right)^{2}+d t^{2}\right\}^{1.5}} d x_{1} d x_{2}
\end{aligned}
$$




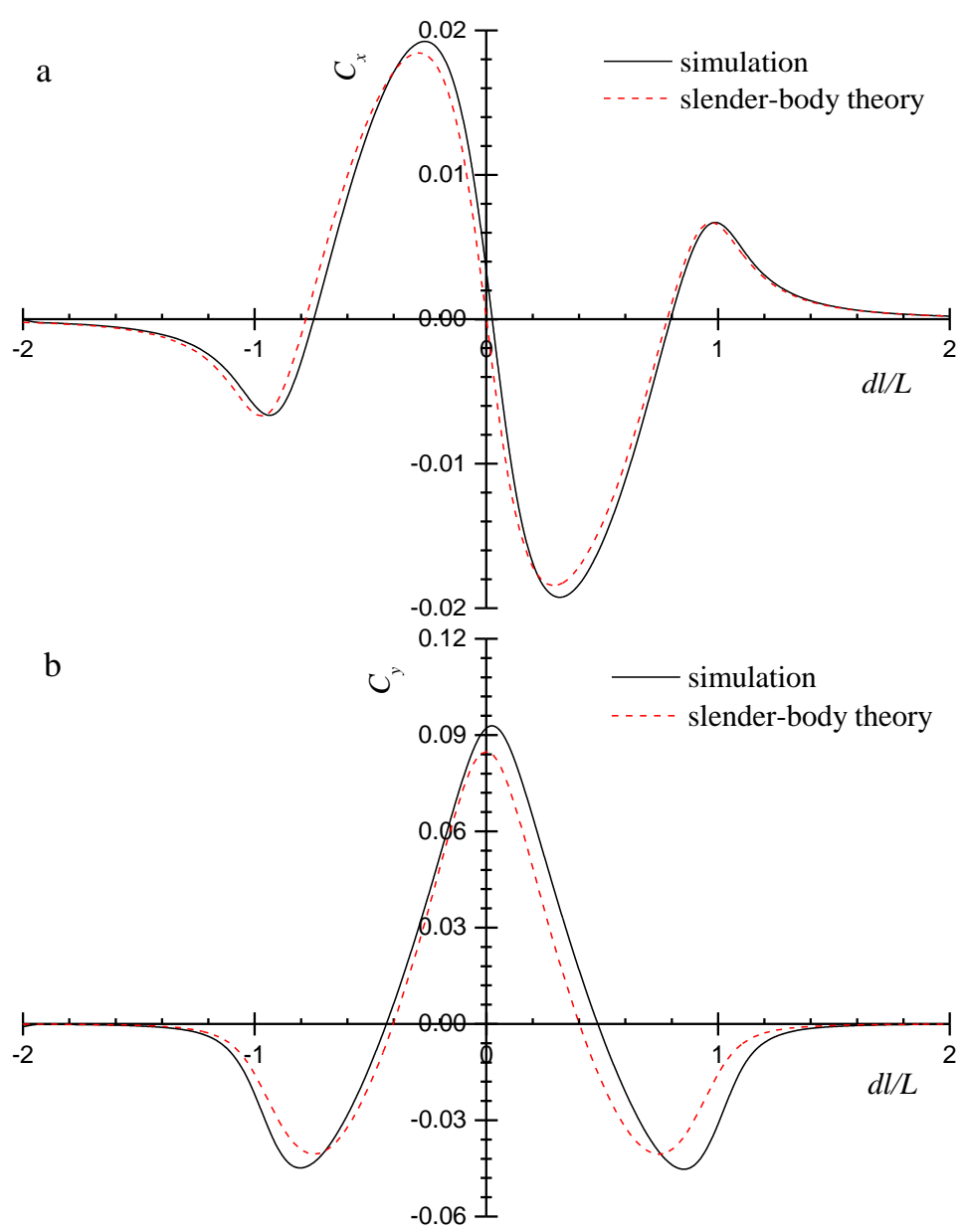

Fig. 6. Time series of hydrodynamic forces acting on the moored ship, $d t=1.5 B, u_{1}=3.589 \mathrm{~m} / \mathrm{s}, u_{2}=0 \mathrm{~m} / \mathrm{s}$. (a) longitudinal force; (b) lateral force.

The hydrodynamic forces obtained with the coupled model and Eq. (21) are compared in Fig. 6. In general, good agreement is acquired. According to Eq. (21), the results predicted by the slender-body theory are strictly symmetric with respect to $d l / L=0$. It is because the free surface elevation effect is neglected and the free surface is treated as rigid boundary in [27]. Comparatively, the free surface elevation is taken into account in this study, leading to the slight discrepancies between the results obtained with the two methods.

\subsection{Validation against model test}

The benchmark model test conducted by Vantorre et al. [8] is used. The two ships involved in the model test were two Esso Osaka models with Froude scale factor 1/75. The model-scale particulars of the two ships are listed in Table 2. The transverse distance between the two ships was

$272 B_{1}+0.5 B_{2}$. Overtaken Ship_1 was translating with speed $0.238 \mathrm{~m} / \mathrm{s}$ while overtaking Ship_2 was travelling at $-0.476 \mathrm{~m} / \mathrm{s}$. 
Table 2 Main particulars of the two ships involved.

\begin{tabular}{lll}
\hline & Ship_1 & Ship_2 \\
\hline Length & $L_{1}=3.824 \mathrm{~m}$ & $L_{2}=3.864 \mathrm{~m}$ \\
Breath & $B_{1}=0.624 \mathrm{~m}$ & $B_{2}=0.55 \mathrm{~m}$ \\
Draft & $D_{1}=0.207 \mathrm{~m}$ & $D_{2}=0.18 \mathrm{~m}$ \\
Block coefficient & $C_{B 1}=0.816$ & $C_{B 2}=0.588$ \\
\hline
\end{tabular}

7. Discrepancies are observed and the numerical model tends to overestimate the wave forces. In

277 the model test environment, the viscous components of the fluid are not negligible and flow

278 separation may also happen. These factors, which can't be considered within the framework of 279 potential theory, dissipate the wave energy and thus the simulated wave forces will be larger.

280 Another cause of the discrepancies is that the complex rudder and the propeller are not established 281 in the coupled model. In spite of the slight discrepancies, the calculation accuracy is acceptable. 

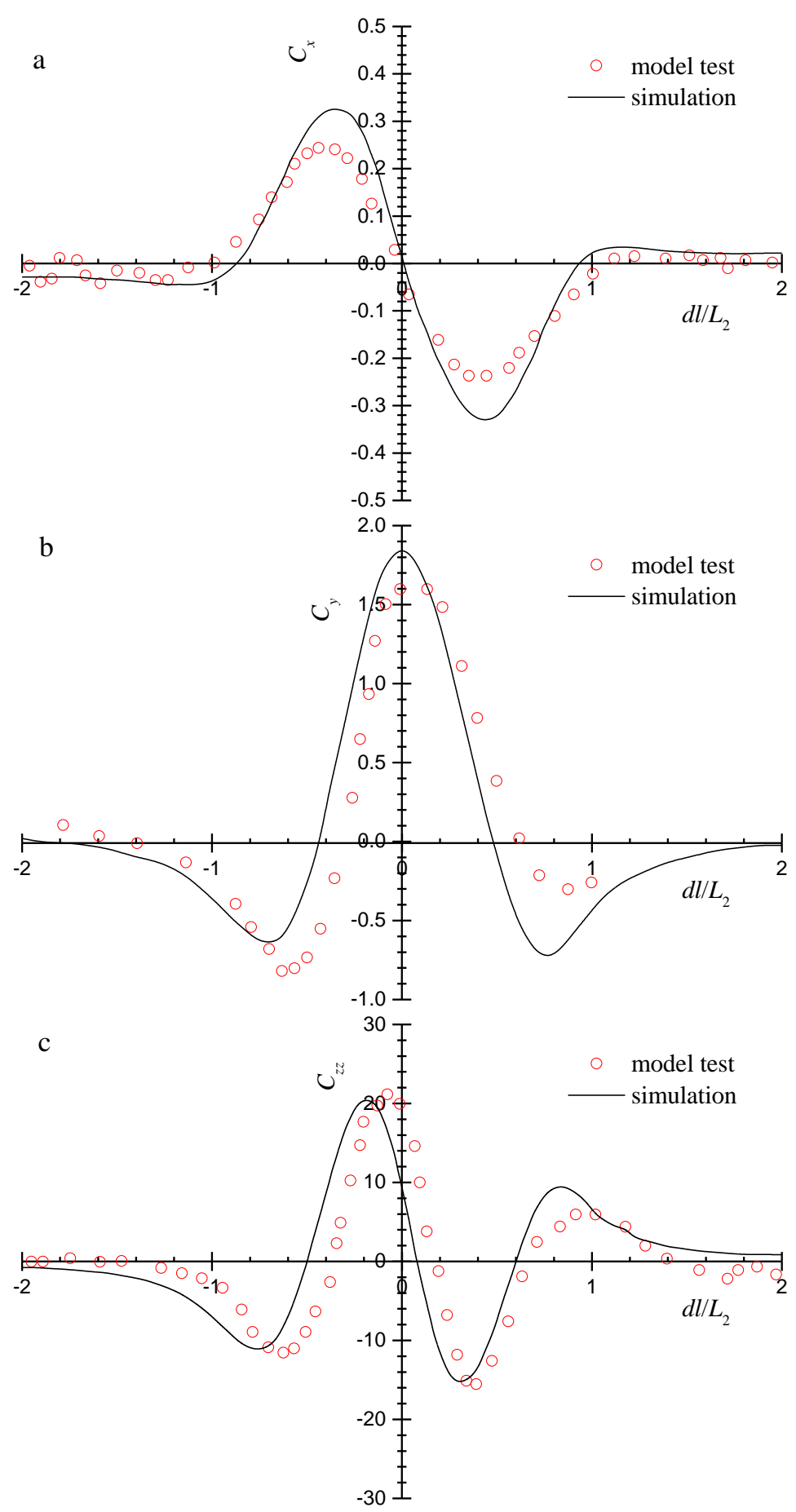

Fig. 7. Time series of hydrodynamic forces acting on Ship_2. (a) longitudinal force; (b) lateral force; (c) yaw moment.

\section{Convergence study}

Fig. 8 illustrates the ship-piers interaction during the passing process. The ship is enforced to pass two side-by-side arranged piers with a constant forward speed $u$ and any oscillating motions are restricted. The main dimensions of the ship are identical to those listed in Table 1. Water depth 


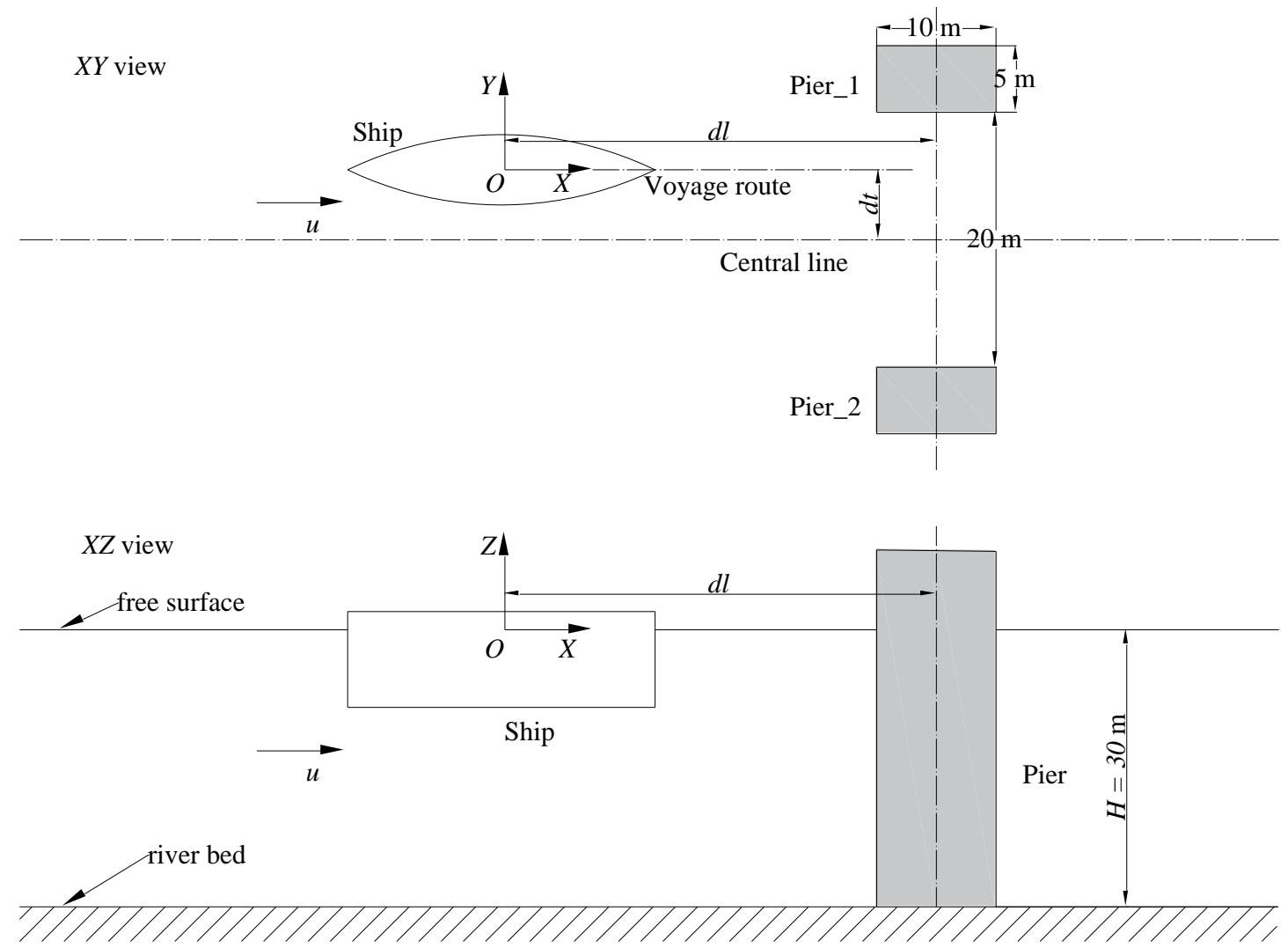

Fig. 8. Illustration of ship-piers interaction during passing process.

Before the numerical simulations are carried out, we first perform the convergence study to seek a suitable configuration of the calculation parameter. Two aspects of the convergence study are addressed, namely the mesh convergence and the time step convergence.

\subsection{Mesh convergence}

The mesh convergence study is firstly performed. Two sets of meshes are generated, namely the standard mesh and the fine mesh. The element size of the fine mesh $(L / d x=60, d x$ is the length of the mesh) is half of that of the standard mesh $(L / d x=30)$. The predicted wave forces obtained with the two mesh configurations are compared in Fig. 9. As shown, the two meshes produce very similar estimation of the hydrodynamic interaction between the passing ship and the piers. Consequently, the standard mesh is capable of producing accurate simulation results. 

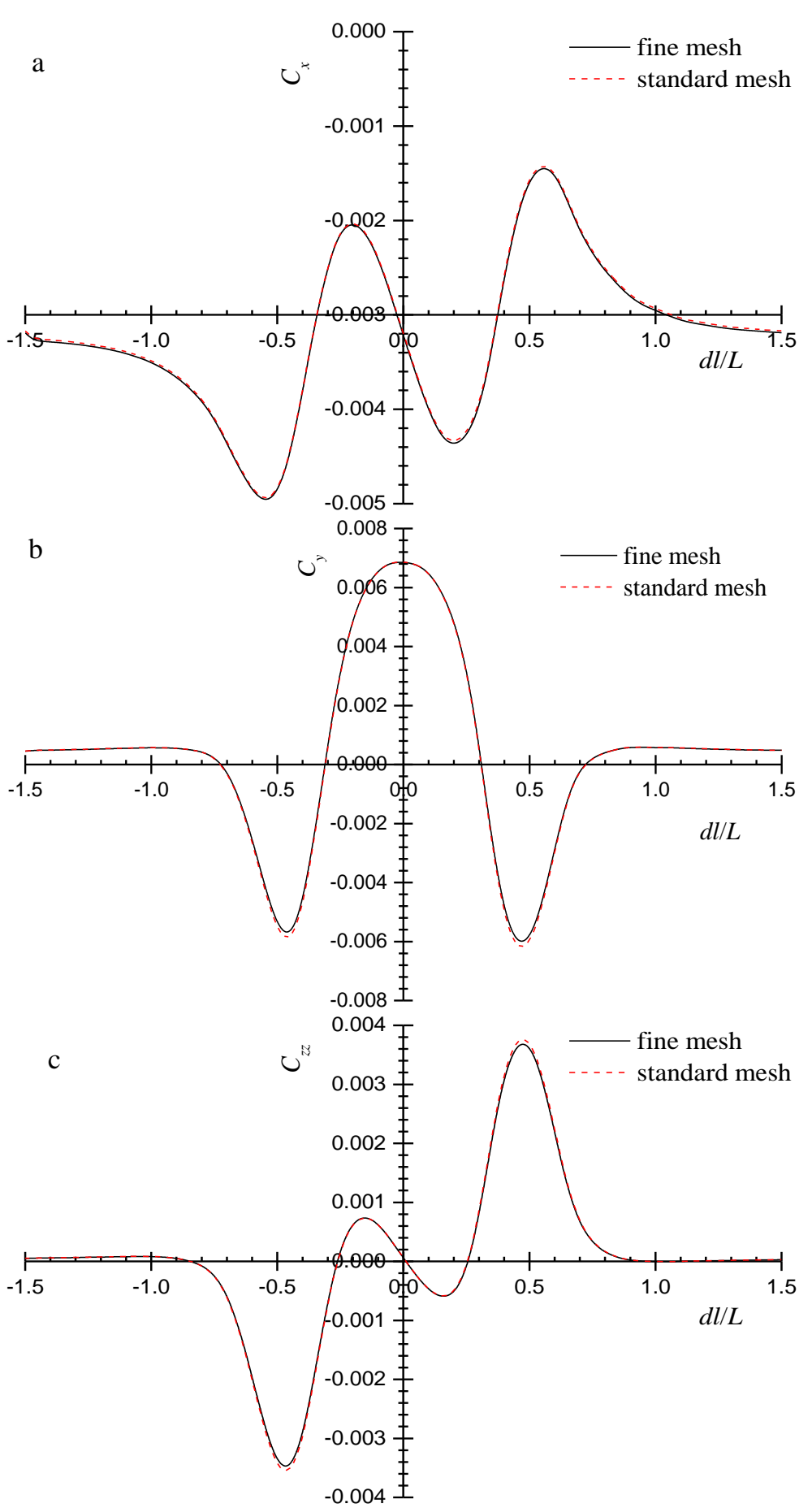

Fig. 9. Mesh convergence study. (a) longitudinal force; (b) lateral force; (c) yaw moment.

\subsection{Time step convergence}

The time step convergence is performed because ship passing piers is by nature a moving boundary problem. It is why the boundary value formula is solved at each time step alongside with update of the free surface element. If the time step is too large, then the calculation may become unstable and some critical time may be missed. We set two sets of time step, namely the standard one $(d t=d x / u)$ and the improved one $(d t=d x / 2 u)$. The comparison of the two time step 
configurations are presented in Fig. 10. The convergence of simulation results is satisfactory.

a

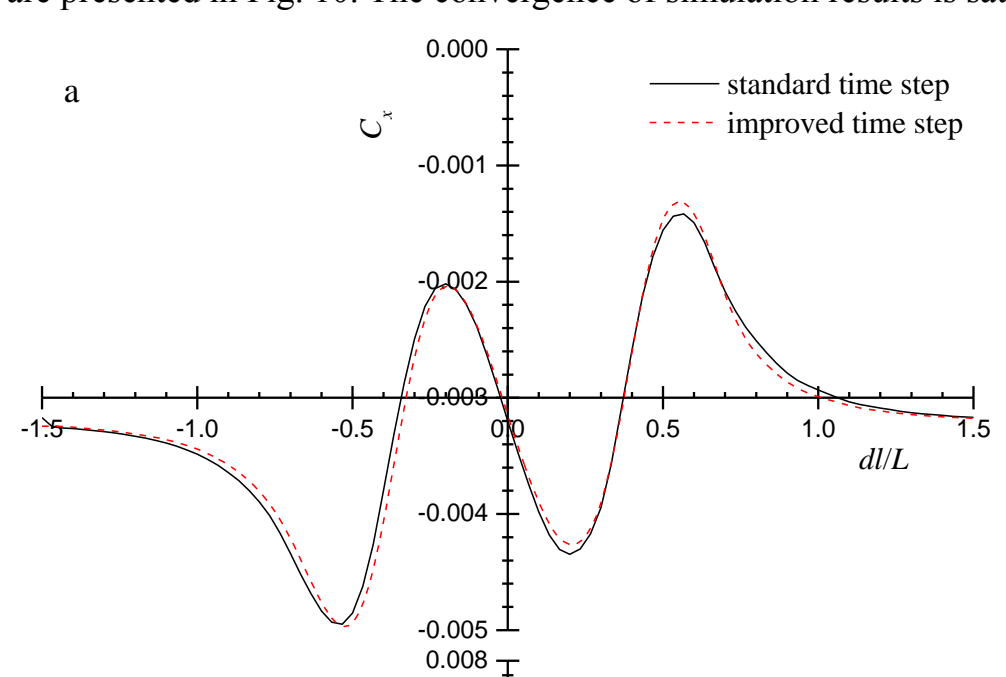

$\mathrm{b}$

314 satisfactory calculation accuracy. Therefore, the standard configurations of mesh and time step will be employed in the following part of this paper. 


\section{Simulation results and discussions}

The three boundary value models discussed in Section 2.1 will be compared in this section. Table 3 gives a brief comparison of the three models.

1. Rigid surface model. The rigid surface model treats the free water surface as a rigid wall. It neglects the wave elevation effect but accounts for the fluid disturbance induced by the piers and the passing ship.

2. Simplified model. The simplified model considers the wave elevation effect but ignores the fluid disturbance induced by the piers.

3. Coupled model. The coupled model proposed in this paper accounts for the wave elevation effect. Furthermore, the fluid disturbance induced by the river flow passing the piers is also considered.

As presented in Section 2.1, one individual boundary value formula is generated at each time step using the rigid model and the simplified model. On the contrary, the velocity potential is divided into two components in the coupled model, and two boundary value formulas are generated. Consequently, the computational cost of the coupled model is generally twice that of the other two models. It is a disadvantage of the coupled model.

Table 3 Comparison of the three models

\begin{tabular}{ccccc}
\hline & $\begin{array}{c}\text { Disturbance } \\
\text { induced by ship }\end{array}$ & $\begin{array}{c}\text { Disturbance } \\
\text { induced by pier }\end{array}$ & $\begin{array}{c}\text { Free water } \\
\text { surface elevation }\end{array}$ & $\begin{array}{c}\text { Computational } \\
\text { cost }\end{array}$ \\
\hline $\begin{array}{c}\text { Rigid model } \\
\begin{array}{c}\text { Simplified } \\
\text { model }\end{array}\end{array}$ & $\sqrt{ }$ & $\sqrt{ }$ & $\times$ & Low \\
$\begin{array}{c}\text { Coupled } \\
\text { model }\end{array}$ & $\sqrt{ }$ & $\sqrt{ }$ & $\sqrt{ }$ & Low \\
\hline
\end{tabular}

Three passing by scenarios are defined: 1) single ship passing piers in calm water; 2) single ship passing piers in river flow; 3) two ships passing piers along opposite direction in calm water. Based on the three numerical models, the unsteady hydrodynamic interactions in the three passing scenarios will be simulated.

\subsection{Single ship passing piers in calm water}

Since the river flow speed is zero, the simplified model and the coupled model are identical to 
each other. Consequently, only the rigid surface model and the coupled model will be compared. It will examine how the wave elevation influences the unsteady hydrodynamic interaction during the passing process.

Fig. 11 displays the time series of wave forces acting on the ship when it is passing the piers with different forward speeds. Despite that the rigid surface model underestimates the peak values of the wave forces slightly, the two numerical models generally produce similar estimations of the hydrodynamic interaction in very low Froude number $(F n=u / \sqrt{g L})$ condition. In Fig. 11 (a), the lateral force is characterized by an initial attraction, followed by repulsion and finally attraction again. It is a desirable phenomenon since the attraction plays a role of restoring force, pulling the ship back to the central line in case of a deviation distance. However, the hydrodynamic interaction tends to push the passing ship toward the piers within region $-0.3<d l / L<0.3$. It is very likely that the shipboard rushes to the piers before exiting this region. From the time series of lateral force, several critical positions for the peak values of the wave forces are identified. The attraction reaches peak value at $d l / L=-0.5$ where the bow just reaches the piers. Another critical position is $d l / L=0.5$ and the stern starts to depart from the piers at this location. The last critical point is $d l / L=0$ where the repulsion reaches maximum value and it is the most dangerous position. Four phases can be distinguished from the time series of yaw moment in Fig. 11 (b). The yaw moment plays a role of bow attraction initially. The attractive yaw moment reaches maximum value when the bow just arrives the piers. It indicates that the bow will turn around towards the central line and the ship returns to the initial voyage route. Although a repulsive yaw moment follows in the second phase, it is too small to turn the ship bow back. In the departing stage, an attractive yaw moment is observed again but at a low-value level. Afterwards, the passing ship is subject repulsive yaw moment and the repulsion is maximized when the stern reaches the piers. The consecutively varying yaw moment is a significant threat to the safe voyage of the passing ship. As well-known, a ship possesses no yaw restoring force and therefore even a small external yaw moment can change its voyage route. Based on the time series of yaw moment, the ship voyage route can be expected to swing consecutively if a rudder correction is not applied. In this circumstance, the bow and the stern are very likely to rush to the piers, which may be more destructive than the shipboard-piers collision. 


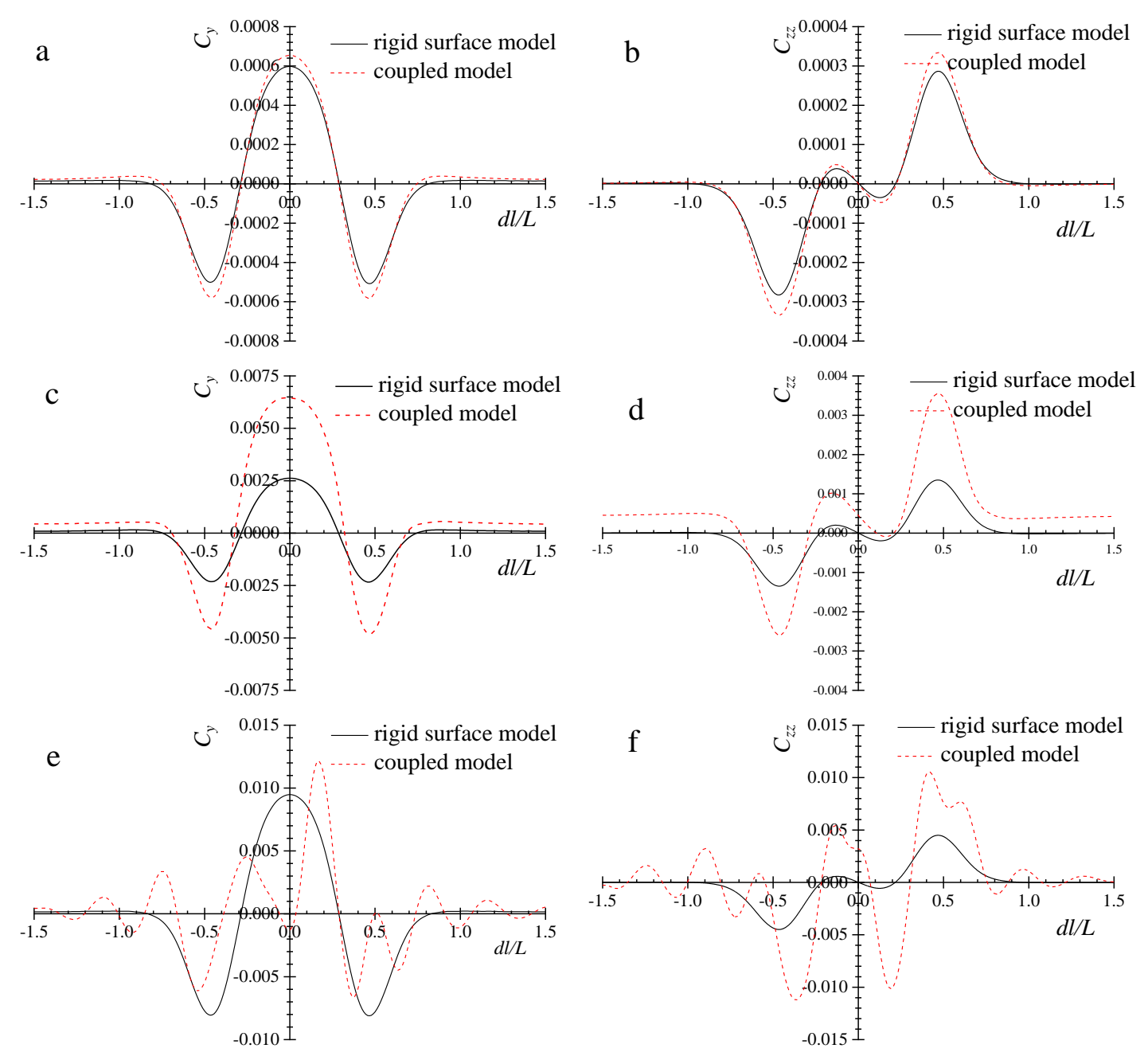

Fig. 11. Time series of wave forces acting on the ship, $d t / B=0.67$. (a) lateral force, $F n=0.06$; (b) yaw moment, $F n$ $=0.06$; (c) lateral force, $F n=0.12$; (d) yaw moment, $F n=0.12$; (e) lateral force, $F n=0.24$; (f) yaw moment, $F n=$

The free surface disturbance is limited in very low forward speed condition and thus the coupled model and the rigid surface model will produce similar results. As the forward speed increases, the discrepancies between the results obtained with the two models become more observable (see Fig. 11 (c) and Fig. 11 (d)). But the characteristics of wave forces remain unchanged. The predicted critical positions are identical to those in the very low forward speed condition. When the forward speed is moderate $(F n=0.24)$, the rigid surface model and the coupled model produce quite different estimations of the unsteady wave forces. In Fig. 11 (e) and Fig. 11 (f), the wave forces obtained with the rigid surface model is still symmetric with respect to $d l / L=0$ and only the peak values are augmented. On the contrary, the results obtained with the coupled model are unstable and multi-phases can be observed throughout the passing process. 

frame, the piers will experience the local wave disturbance region, the divergent wave disturbance region and finally the transverse wave disturbance region during the whole passing process (see Fig. 12). The Kelvin wake is negligible at very low forward speed and it is local wave that dominates the hydrodynamic interaction between the passing ship and the piers. In this circumstance, the hydrodynamic interaction is mainly dependent on longitudinal distance between the ship and the piers. It explains the symmetric property with respect to $d l / L=0$. On the contrary, the Kelvin wake is significant at moderate Froude number and the discrepancies between the three wave regions are observable. Consequently, the hydrodynamic interactions during approaching stage and departing stage are different.
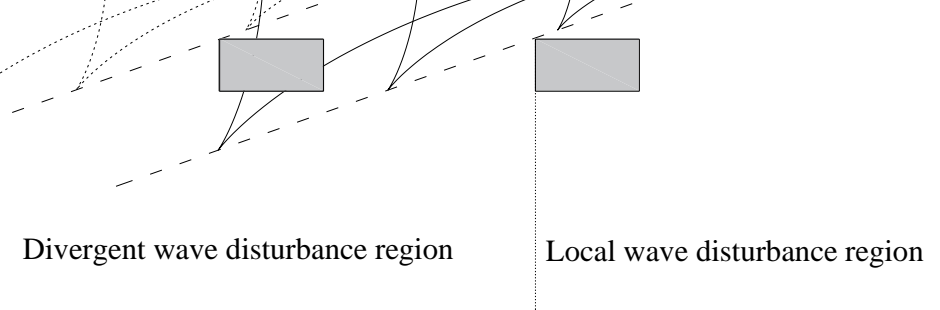

Fig. 12. Sketch of Kelvin wake.

Fig. 13 displays the wave patterns when the ship is passing by the piers with moderate forward speed. When the ship is approaching to the piers in Fig. 13(a), the piers are outside the Kelvin wake region so the local wave disturbance is the essential factor. Whereas in Fig. 13(b), the piers are located within the ship's wake. Due to the presence of piers, the divergent wave is not free to propagate outside. It is obvious that the wave elevation effect is significant so that the rigid surface model is not valid. 

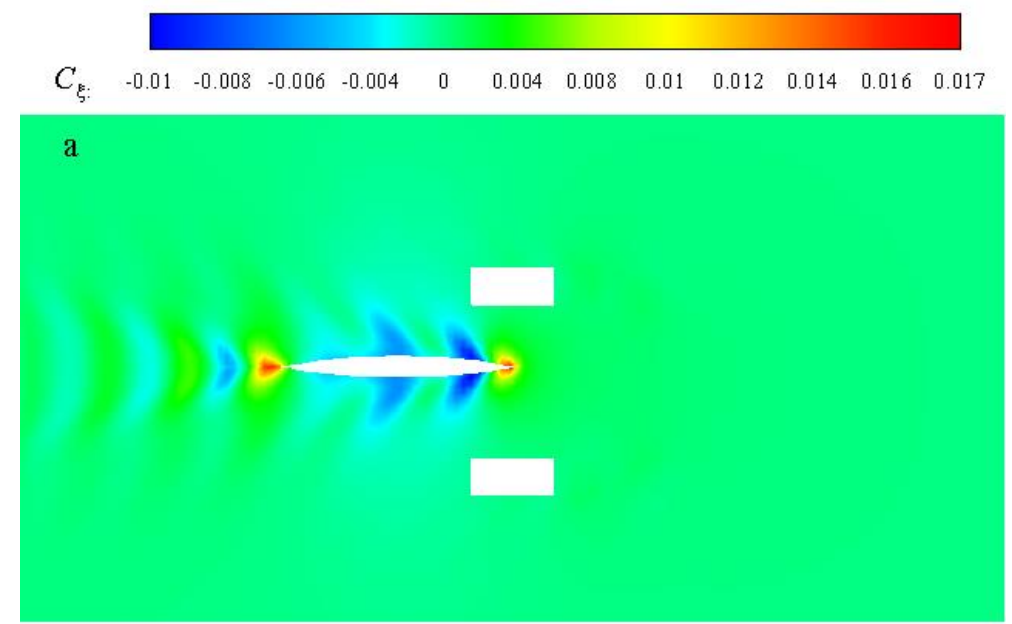

b

400

Fig. 13. Wave patterns at $F n=0.24, C \xi=\xi g / 2 \pi u^{2}$. (a) $d l / L=-0.5$; (b) $d l / L=0.5$.

\subsection{Single ship passing piers in river flow}

Fig. 14 displays the time series of wave forces acting on the ship throughout the passing process. Regardless of the numerical models used, the lateral wave force is characterized by an initial attraction, followed by repulsion and finally attraction again. This variation characteristic is consistent with that in calm water condition. Despite this common characteristic, the three numerical models produce distinct estimations of the lateral force. In general, the rigid surface model and the coupled model produce similar simulation results, although the rigid surface model underestimates the peak values of later force. Both the rigid surface model and the coupled model predict that the maximum attraction occurs at $d l / L=-0.7$ and $d l / L=0.7$, respectively. Nevertheless, the results predicted by the rigid surface model are completely symmetric with respect to $d l / L=0$ whereas the results obtained with the coupled model is not. The cause of such discrepancy has been clarified in Section 5.1 .

Although the results obtained with the simplified model and the coupled model show similar characteristics, the simplified model not only underestimates the lateral force significantly, but also 
predicts different critical positions for the peak values of lateral force. As shown in Fig. 14 (a), the maximum attraction is observed at $d l / L=-0.4$ and $d l / L=0.45$ when the simplified model is used.

418 Similar conclusion can be drawn from the yaw moment displayed in Fig. 14 (b). The time series of yaw moment is characterized by four phases and four peak values. The critical positions for peak values predicted by the rigid surface model and the coupled model are identical. On the contrary, the simplified model predicts different critical positions.
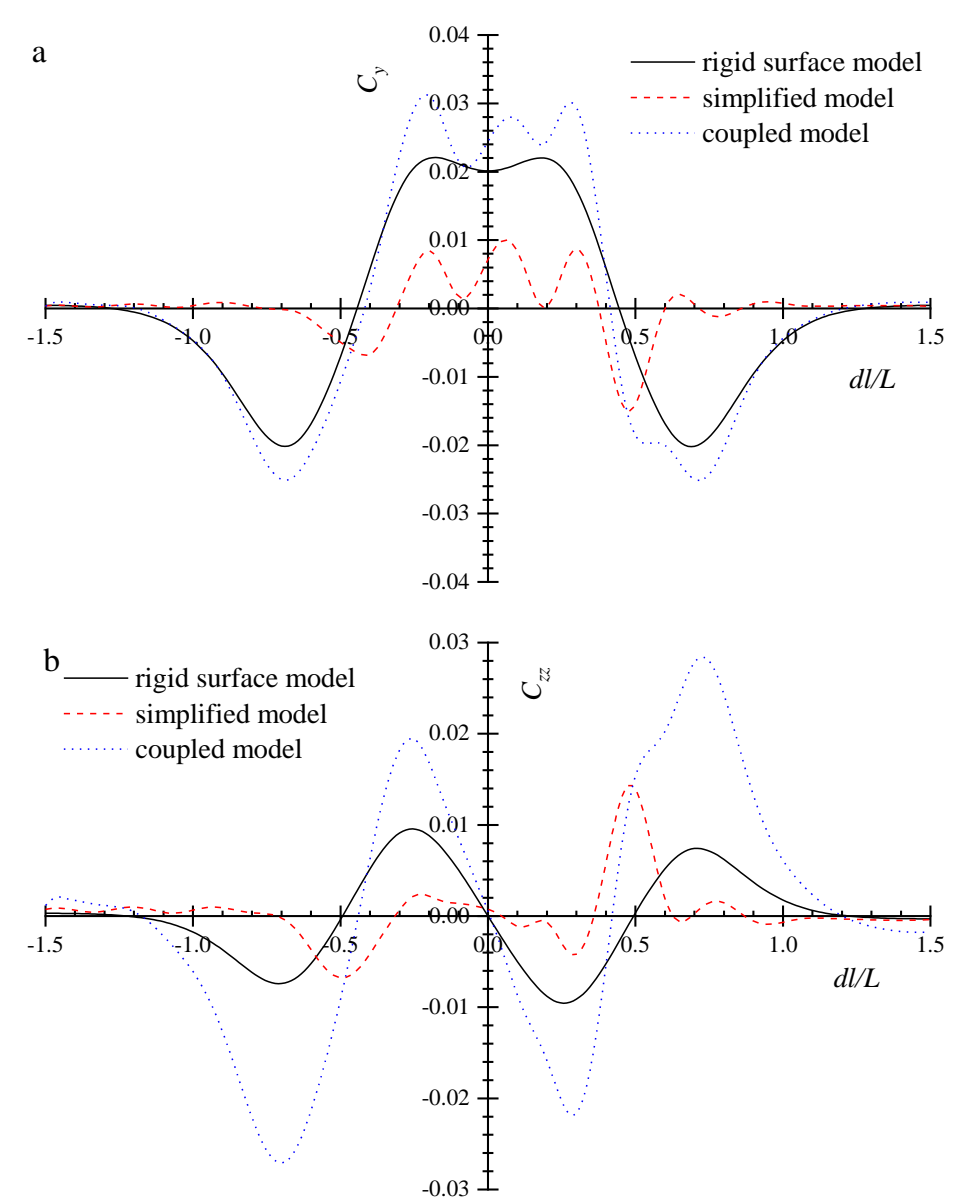

Fig. 14. Time series of wave forces acting on the ship; $F n_{-}$ship $=0.24, F n \_$river $=0.03, d t / B=0.67$. (a) lateral force; (b) yaw moment.

Simulation results show that the piers-induced fluid disturbance is vital to the ship-piers hydrodynamic interaction even if the river speed is small. It is probably attributed to the fat shape of the piers. According to the simulation results, ship passing piers in a river must be addressed as a different forward speeds problem.

\subsection{Two ships passing piers in calm water}

In this section, two Wigley ships are enforced to pass the piers along opposite direction (see 
431 Fig. 15). The forward speeds of the two ships are identical so that they will meet each other exactly at the piers.

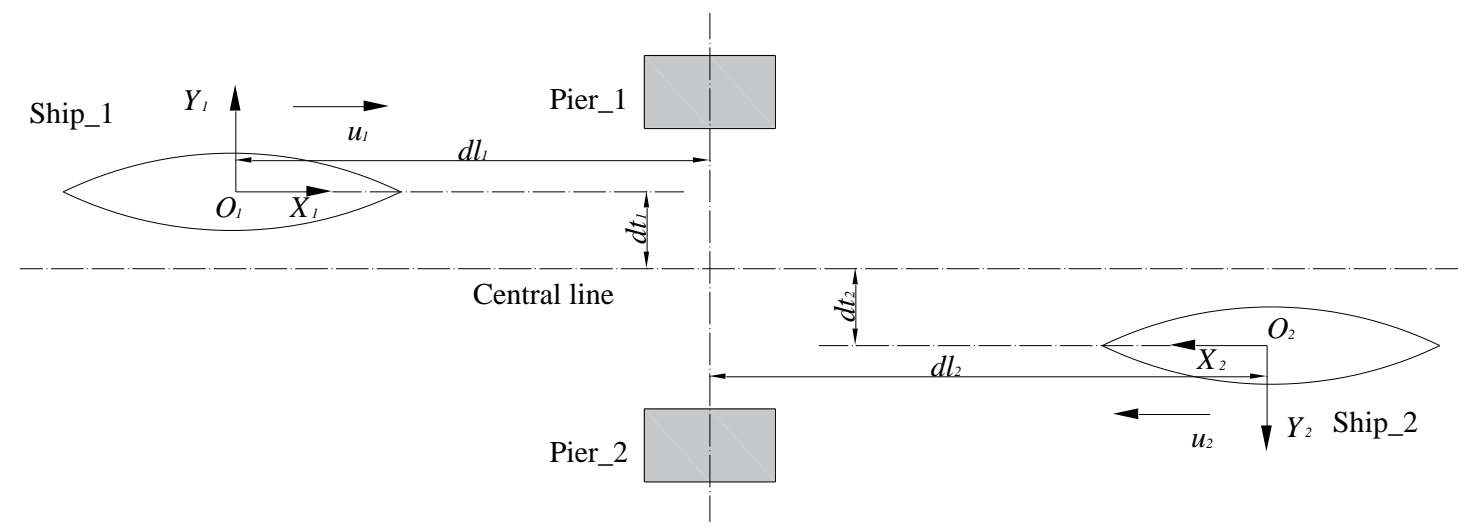

Fig. 15. Sketch of two ships passing piers along opposite direction.

The time series of unsteady wave forces acting on ship_1 is displayed in Fig. 16. When the two ships are approaching to each other with very low forward speed, the rigid surface model and the coupled model produce very similar estimations of the wave forces. The lateral force is characterized by initial repulsion, followed by attraction and finally repulsion. Three extreme values of the wave force are observed at $d l / L=-0.4, d l / L=0$ and $d l / L=0.4$. It is an interesting finding that the variation characteristic is opposite to that in Fig. 11. As well known, two moving ships will attract each other during their encountering maneuvering. Therefore, it is concluded that ship-toship interaction, rather than ship-to-piers interaction, plays the dominating role in this scenario. Three phases and three extreme values are identified in the times series of yaw moment. The yaw moment is characterized by attraction initially, followed by repulsion and attraction again. When the two ships are very close, they tend to swing away from each other under the hydrodynamic interaction to avoid the ship-ship collision. 

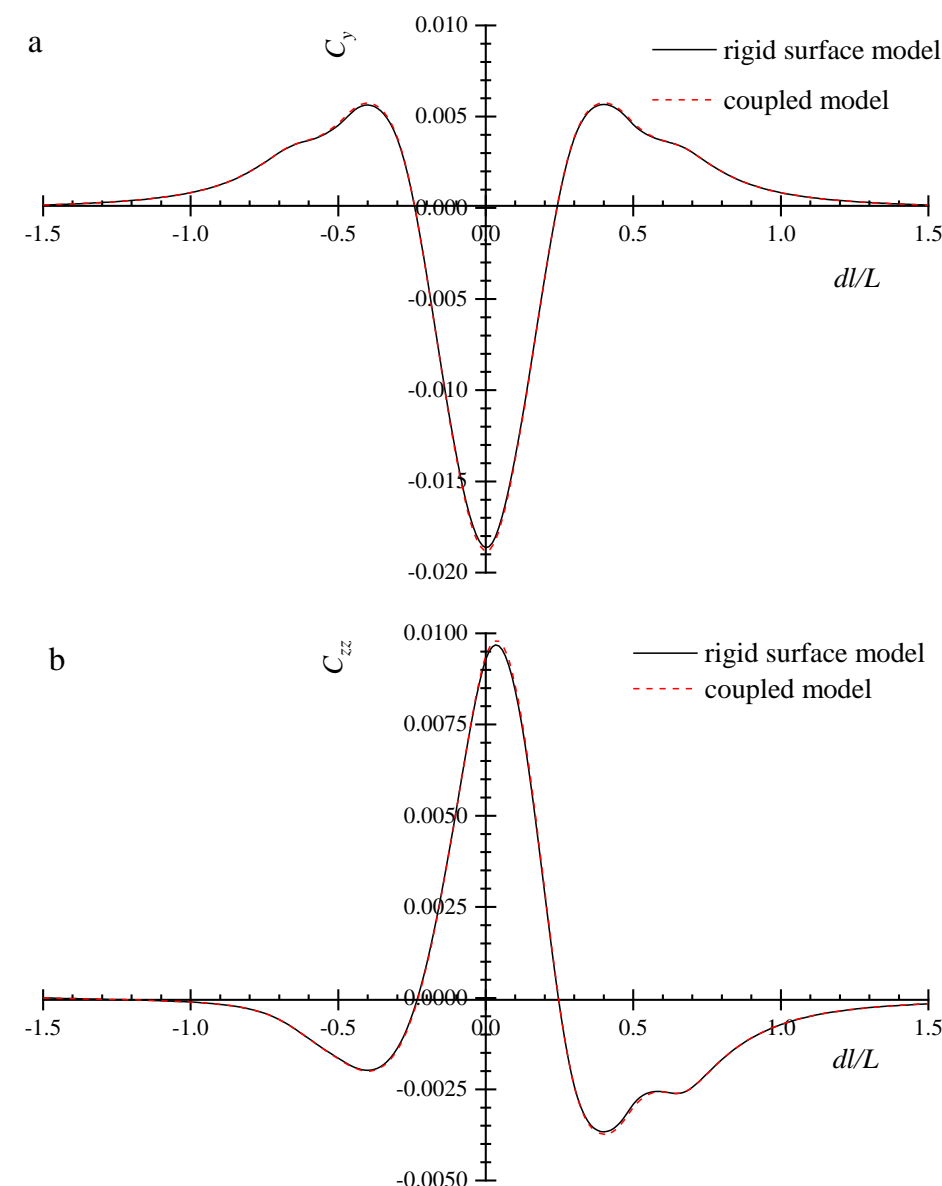

Fig. 16. Time series of wave forces acting on Ship_1, Fn_ship_1 $=0.06, F n \_$ship_ $2=0.06$. (a) lateral force; (b) yaw moment.

Fig. 17 displays the wave forces when the two ships are passing the piers with moderate forward speed, in which the results obtained with the two models are distinguished from each other. Three phases can be identified in the results predicted by rigid surface model and the wave forces are generally symmetric with respect to $d l / L=0$. On the contrary, the hydrodynamic interactions during the approaching and departing stages are quite distinct when the coupled model is used. In the departing stage, the hydrodynamic interaction is unstable and multi-phases are observed. It is a serious challenge to the safe voyage of passing ships since the varying wave forces will induce strong sway and yaw motions. Fig. 18 shows the wave patterns before and after the encountering. Since the forward speed is moderate, the free surface elevation is noticeable and V-shape wake is observed. During the approaching stage, Ship_1 is not influenced by wake induced by Ship_2 and vice versa (see Fig. 18 (a)). It explains why the lateral force is relatively stable before the encountering. On the contrary, the Ship_1 is in the wake induced by Ship_2 and experiences wave 
462 crest and trough alternately. It induces the high frequency component of wave force in depart stage.

463 Meanwhile, the envelope curve drops gradually indicting that ship-piers interaction becomes weaker 464 with the increase of longitudinal distance.
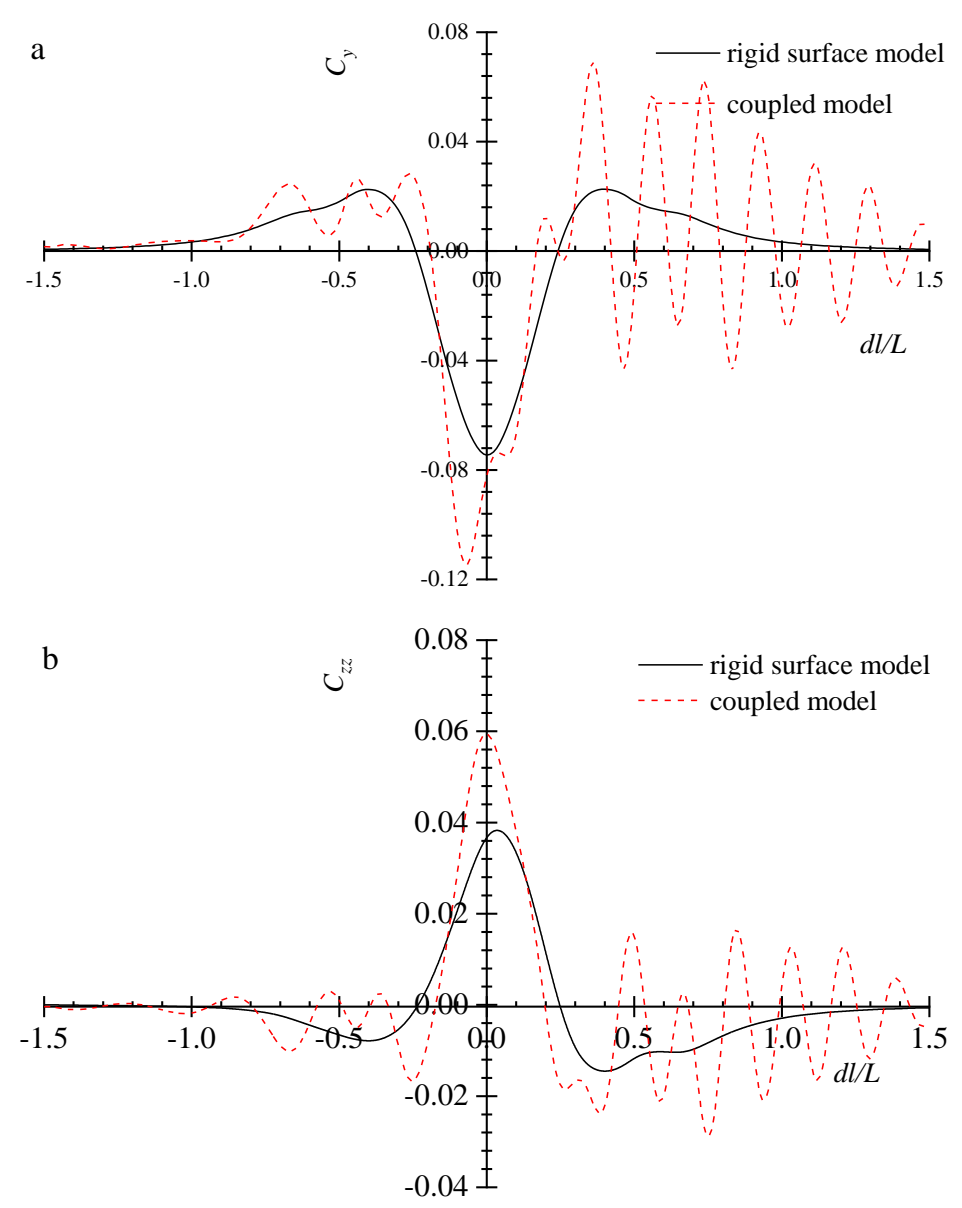

Fig. 17. Time series of wave forces acting on Ship_1, Fn_ship_ $1=0.24, F n \_$ship $\_2=0.24$. (a) lateral force; (b) yaw moment. 

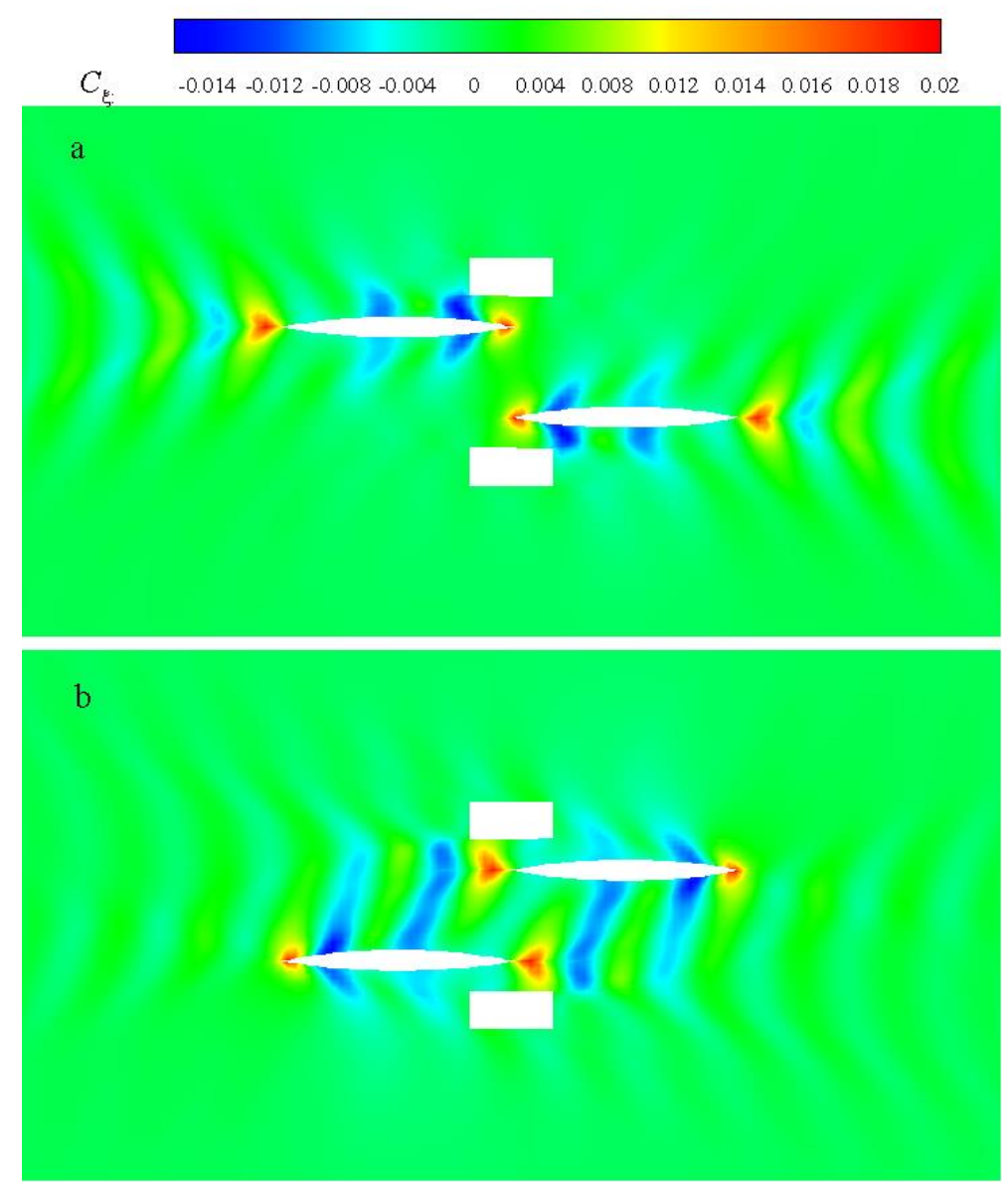

Fig. 18. Wave patterns at $F n \_s h i p \_1=0.24, F n \_s h i p \_2=0.24, C \xi=\xi \mathrm{g} / 2 \pi u_{1} u_{2}$. (a) $d l / L=-0.5$; (b) $d l / L=0.5$.

\section{Conclusions}

A 3-D boundary element method based on the Rankine type Green function is developed to investigate the ship-piers hydrodynamic interaction during passing process. A re-meshing algorithm, based on local mesh and global mesh concepts, is proposed to update the truncated free surface at each time step. The different forward speeds problem is addressed with an uncoupled method, which divides the velocity potential into two separate components. Three numerical models for ship passing piers problem are compared: 1) the rigid surface model which neglects the wave elevation effect; 2) the simplified model which neglects the piers-induced fluid disturbance; 3) the coupled model which accounts for both the wave elevation effect and the piers-induced fluid disturbance. Based on the three numerical models, the unsteady hydrodynamic interactions between the passing ship and the piers are simulated. 
that the forward speed is very low. Compared with the coupled model proposed in this study, the rigid surface model can enhance the calculation efficiency since just a single boundary value equation is established regardless of the number of bodies involved. Such advantage will become more observable when a couple of bodies are involved in the hydrodynamic interaction. For low forward speed problems where the Froude number is less than 0.12 , the rigid model is recommended. Although the simplified model considers the wave elevation effect in the estimation of shippiers hydrodynamic interaction, its prediction capacity is unsatisfactory. It inherently indicates that the fluid disturbance induced by the piers is vital to the ship-piers interaction even if the river speed is low compared with ship forward speed. Although the computation efficiency of the simplified model is desirable, it is not recommended for application in passing-pier passing problem.

Through the comparisons between the free numerical models, it is proved that the ship passing piers problem must be addressed as an encountering problem. In most cases, the wave elevation effect should be considered, and the coupled model is recommended.

\section{Limitation and future work}

Although the coupled model proposed in this paper performs better than the other two models, by considering the free surface disturbance and the flow induced by the piers, it is by nature a quasisteady method. In high speed encountering and overtaking problem, the unsteadiness and the nonlinearity involved in the hydrodynamic interaction becomes increasingly important. As a result, the present methodology may be invalid. Future work will concentrate on this aspect and a fully unsteady method should be developed.

\section{Acknowledgement}

This work was supported by China Scholarship Council [Grant No. 201506230127]. The authors are grateful for their financial support.

\section{References}


508 [2] Mancini G, Malfatti A. Full scale measurements on high speed train Etr 500 passing in open air and 509 in tunnels of Italian high speed line. Note N Fl Mech Mul D 2002;79: 101-22.

510 [3] Watanabe K, Matsuno K. Moving computational domain method and its application to flow around 511 a high-speed car passing through a hairpin curve. Journal of computational Science and Technology $512 \quad 2009 ; 3(2): 449-59$.

513 [4] Yeung RW, Tan WT. Hydrodynamic interactions of ships with fixed obstacles. J Ship Res 1980;24(1).

514 [5] Kijima K. Prediction method for ship manoeuvring motion in the proximity of a pier. Ship Technology $515 \quad$ Research 1997;44(1): 22-31.

516 [6] Newton R. Some notes on interaction effects between ships close aboard in deep water. Proc. 1st 517 Symposium on Ship Maneuverability 1960;1-24.

518 [7] Müller E. Untersuchungen über die gegenseitige Kursbeeinflussung von Schiffen auf 519 Binnenwasserstrassen. Schiff und Hafen 1967;19(6): 393-406.

520 [8] Vantorre M, Verzhbitskaya E, Laforce E. Model test based formulations of ship-ship interaction forces. $521 \quad$ Ship Technology Research 2002;49: 124-41.

522 [9] Mousaviraad SM, Sadat-Hosseini SH, Stern F. Ship-ship interactions in calm water and waves. Part 523 1: Analysis of the experimental data. Ocean Eng 2016;111: 615-26.

524 [10] Tuck E, Newman J. Hydrodynamic interactions between ships. 10th Symposium on Naval 525 Hydrodynamics. Cambridge, Mass 1974. 35-70.

526 [11] Yeung RW. On the interactions of slender ships in shallow water. J Fluid Mech 2006;85(01): 14352759.

528 [12] Brix J. Manoeuvring Technical Manual. Schiff und Hafen 1987;36(5).

529 [13] Wang QX. An analytical solution for two slender bodies of revolution translating in very close 530 proximity. J Fluid Mech 2007;582: 223-51.

531 [14] Xiang X, Faltinsen OM. Time domain simulation of two interacting ships advancing parallel in 532 waves. ASME 2011 30th International Conference on Ocean, Offshore and Arctic Engineering: 533 American Society of Mechanical Engineers; 2011. 357-69.

534 [15] Sutulo S, Soares CG, Otzen JF. Validation of Potential-Flow Estimation of Interaction Forces Acting 535 upon Ship Hulls in Parallel Motion. J Ship Res 2012;56(3): 129-45.

536 [16] Yuan ZM, He S, Kellett P, Incecik A, Turan O, Boulougouris E. Ship-to-Ship Interaction during 
Overtaking Operation in Shallow Water. J Ship Res 2015;59(3): 172-87.

538 [17] Yao CB, Dong WC. Numerical study on local steady flow effects on hydrodynamic interaction 539 between two parallel ships advancing in waves. Eng Anal Bound Elem 2016;66: 129-44.

540 [18] Xu HF, Zou ZJ, Zou L, Liu XY. Unsteady hydrodynamic interaction between two cylindroids in 541 shallow water based on high-order panel method. Eng Anal Bound Elem 2016;70: 134-46.

542 [19] Pinkster J, Bhawsinka K. A real-time simulation technique for ship-ship and ship-port interactions.

543 28th International Workshop on Water Waves and Floating Bodies (IWWWFB 2013), L'Isle sur la Sorgue,

544 France2013.

545 [20] Wang HZ, Zou ZJ. Numerical study on hydrodynamic interaction between a berthed ship and a ship 546 passing through a lock. Ocean Eng 2014;88: 409-25.

547 [21] Newman JN. Linearized wave resistance theory. International Seminar on Wave Resistance. Tokoy, 548 Japan1976. 31-43.

549 [22] Hess JL, Smith A. Calculation of non-lifting potential flow about arbitrary three-dimensional bodies. 550 DTIC Document; 1962.

551 [23] Kim Y, Yue DKP, Connell BSH. Numerical dispersion and damping on steady waves with forward speed. Appl Ocean Res 2005;27(2): 107-25.

$553[24] \mathrm{Xu} \mathrm{H}$, Yue D. Computations of fully nonlinear three dimensional water waves. Proceedings of the 554 19th Symposium On Naval Hydrodynamics. Seoul, Korea1992.

555 [25] Longuet-Higgins MS, Cokelet ED. The deformation of steep surface waves on water. I. A numerical 556 method of computation. Proceedings of the Royal Society of London A: Mathematical, Physical and 557 Engineering Sciences: The Royal Society; 1976. 1-26.

558 [26] Bunnik THJ. Seakeeping calculations for ships, taking into account the non-linear steady waves. 559 Delft, Netherlands: Delft University of Technology; 1999.

560 [27] Varyani KS, Krishnankutty P. Modification of ship hydrodynamic interaction forces and moment by 561 underwater ship geometry. Ocean Eng 2006;33(8-9): 1090-104. 